\title{
Neural network model as a way of processing complex systems of econometric equations characterizing the interaction of the Russian Arctic
}

\author{
Sergey Antipov ${ }^{1, *}$ \\ ${ }^{1}$ Peter the Great St.Petersburg Polytechnic University, Polytechnicheskaya, 29, St. Petersburg, \\ 195251, Russia
}

\begin{abstract}
This article analyses the possibility of applying neural network modeling for the purpose of automation of a large number of calculations of econometric equations coefficients in order to obtain adequate predictive results.
\end{abstract}

\section{Introduction}

Any systematic development is possible only in case the competent strategy has been worked out. The strategy should include many predictions that can take into account not only different features of the researched economic object, but the maximum number of external factors as well. This reasoning can be attributed to the formation of Arctic development strategy. It's obvious, that taking into account all the random effects is impossible.

\section{Literature review}

One should have accurate and reliable models for making a qualitative prediction of the economic development of the Arctic and the profitability analysis. The models should be capable of automated assessment of the current economic situation provided that the maximum number of influencing factors is considered. [1,4]

During the initial assessment and the subsequent analysis one can use either linear or nonlinear equations. In terms of increased accuracy the application of ADL-model may be optimal. The ADL-models are capable of describing not only the direct relationship between exogenous and endogenous variables, but also reflecting the lagged shifts that are very important in the study of econometric variables.

\section{The methodology}

\footnotetext{
${ }^{*}$ Corresponding author: skantipov@gmail.com
} 
In fact, the overall view of the equations of that model may be as follows:

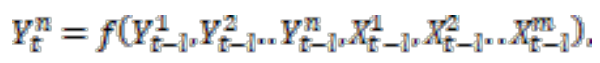

Where:

- $\quad \mathrm{Y}$ - endogenous variables

- $\mathrm{X}$ - exogenous variables,

- $\mathrm{t}$ - time,

- 1 - lag,

- $\mathrm{n}, \mathrm{m}$ - ordinal indices.

From the modeling point of view the inductive approach can be considered as optimal. This approach means that modeling starts with the lowest levels that include private parameters for certain sectors of research; thereafter the second-level models are created. The models of the second level reflect the relationship between the first-level models. So the process continues until the ultimate top-level model is built. Under this analysis the resulting model will directly describe the key aspects of economic development, while in the same it indirectly reflects all of the factors included in the lower-level models. [3, 5-7]

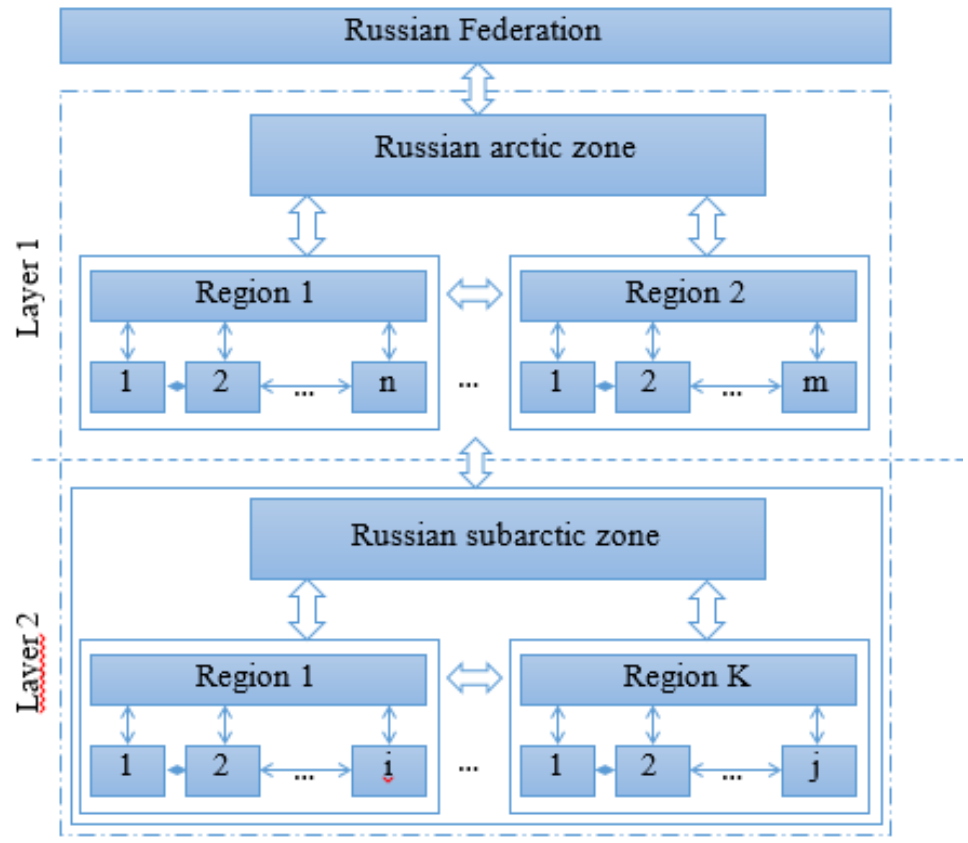

Fig. 1. The block diagram of interaction of arctic and subarctic regions.

In the proposed model the main two layers are the Arctic and the subarctic zones. Each layer is divided into sub-layers, containing the information on the regions and the entities of the specified zones. The arrows represent the interaction flow (information, material, natural, etc.). Both layers are equal with respect to the final principal object which is the State as a whole. The model allows the evaluation of all possible interactions in various combinations and permutations. $[8,9,10]$

While talking about development strategy, we always have in mind the following: having a set of the input data, such as geopolitical status, geographical location, economic situation, development of infrastructure and social sphere etc., one needs to obtain the final 
result a set of instructions for further action that could improve the current situation. So far, initially one can only presume the resulting variable, but with no chance of defining its parameters and resulting value beforehand. In order to define the parameters an ADLequation is used. [11-13]

\section{Model}

In forecasting the economic development of the Russian Arctic it is the most convenient to use a three-tier model (Fig. 1). From Figure 1 it can be seen that the general model should track the multilevel interaction. It's necessary to have an assessment of both the interactions between specific areas, and between the separate regions and actors. Along with this, it is important to have an idea about the contribution of the represented regions to the development of the national economy. It also needs to be reflected in the model. [2]

Developing of the ADL model can be divided into 4 main stages:

1. Stage 1. Determining the key factors that have a maximum impact on the regional economy. At this stage, the economic indicators are selected and statistical data on their dynamics is collected.

2. Stage 2. Identifying the relationship between the selected factors. Determining of the exogenous and the endogenous variables. The selected indicators are checked for linkages with each other. In the simplest scenario, with the system of linear equations, the correlation matrix is constructed and the dependent and influencing factors are determined on the basis of linear estimates.

3. Stage 3. Building of a system of independent ADL equations. Based on the relationships obtained at the second stage, a system of equations that algorithmically reflect these relationships is built for each region. A key feature of ADL model is not just a reflection of the relationship between the variables in certain time points, but also the relationship with a time lag.

4. Stage 4. Determination of interregional interaction. Obtaining the final system of equations that reflects the relationship between the regions in terms of the dependent variables.

Ultimately on the each tier there will be a system of interrelated equations of the form:

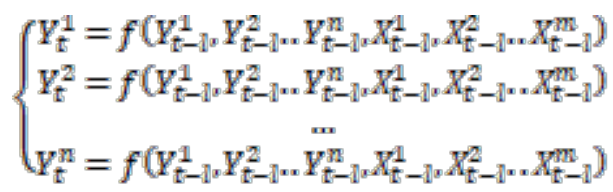

If we consider the models of the lower levels, there will be plenty of systems of equations, given that it is expected to give a detailed description of each area of economic activity in each region. [3] The solution for all the systems will lead to an extremely accurate understanding of economic activities of the regions, their interactions, their foreign trade activities, as well as the impact on the Russian economy as a whole.

However, calculation of such a great number of parameters will be too time consuming and won't always give the up-to-date analytical data. In this regard, there arises a problem of optimization of the calculations concerning the systems of equations. For these purposes the neural networks come to use.

The overall essence of neural network modeling is quite simple and is logically understandable. The idea is that there are a number of input parameters and the task is to identify some output parameters. To implement this correlation the neural model defines such notion as weight - some ratio, indicating the impact of some input parameter in the resulting output variable. 


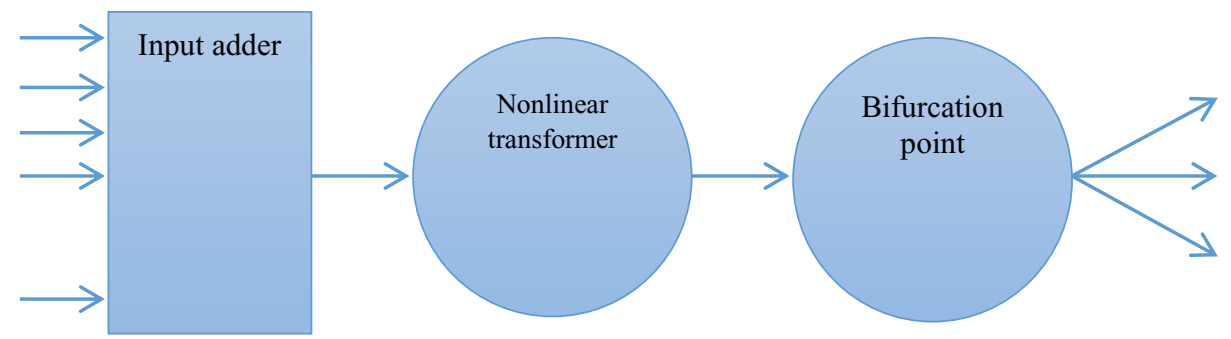

Fig. 2. General view of the neural network model.

In Figure 2 the input adder receives input data, thereafter the weights of these parameters are calculated by nonlinear transformations, and after the bifurcation point the results are obtained as the output data. Thus, all the relationships between the input and the output values are fully described. Changing the sets of model parameters one can change the model and track the development of various patterns.

The main feature of neural networks is that the relationship between input and output is the matter of learning of the network. It means that with new input data the network itself will be changing on the basis of the values of its elements and, therefore, the result will also change. The more input parameters we have, the more precise are the calculations of the transfer functions and the more accurate is the final result. The models based on neural networks are one of the few, for which a great number of variables and input values is not a problem, but rather a serious advantage.

Returning to the task of determining the parameters of interconnected systems of equations, the input data will be the economic indicators selected by the researcher. The nonlinear transformer will be the computer system that will determine the weights (coefficients) of the equations as the output result obtained after the bifurcation point.

Considering the functioning of the proposed neural model, one can split it into several stages. At the first stage, after the input indicators has been fed in the system, the algorithm analyses the data and evaluates the extent of their interdependence. The endogenous and exogenous variables are being identified. At the second stage the form relationship between endogenous and exogenous variables is being determined so that the optimal type of equation can be selected. At the third stage the coefficients are calculated for the selected equation type. The fifth stage, the last one, finishes the calculation algorithm of neural network by gathering all the coefficients. At this stage the final quantitative model is shaped. The proposed method provides also a feedback that will allow customizing of calculations on each stage in order to enable the learning process within the network as well as improving the final result by correction of either the input parameters or the computational techniques. [1]

In this method the neural model is used as an auxiliary element of both the selection of interdependent variables, and the calculation of the coefficients of equations. This complex approach, i.e. the synthesis of ADL model and neural network modeling will simplify computing tasks for the researcher and will always reflect the most current information by accelerating the calculation process. It will also enable to observe changes at all levels of the model in the real-time mode.

On the basis of such complex model one can give not only the estimated values of the output functions, but also quite accurate results of the output parameters, which make the further conclusions possible. Having interpreted and analyzed the results correctly, one can get the precise and clear instruction for the formation of either economic or political development strategy. 
While creating any model one should always bear in mind that all decisions on formation of development strategies will depend entirely on the person, since even the most advanced computing engine won't give the correct answer to the question "What to do?". Essentially the results there are no more than guidelines and vectors for the research, starting points for decision-making. But the more precise and accurate are these starting points, the more likely the right choice will happen.

\section{Conclusions}

Actually it is practically impossible to create a strategy for a long term in an unstable economic environment. Moreover, the model will need some adjustments due to changing external factors and various influences. Therefore, when designing a model, one should envisage the possibility of data correction within the model. It is important to understand that not only sets of input data may be adjusted, but the number of input parameters, the input parameters themselves and the forms of identified patterns as well.

Based on the stated above, it can be noted that creation of recurrent neural network model seems the most useful. The model is based on the proposal of the Figure 1, however with some correcting additions (Fig. 3).

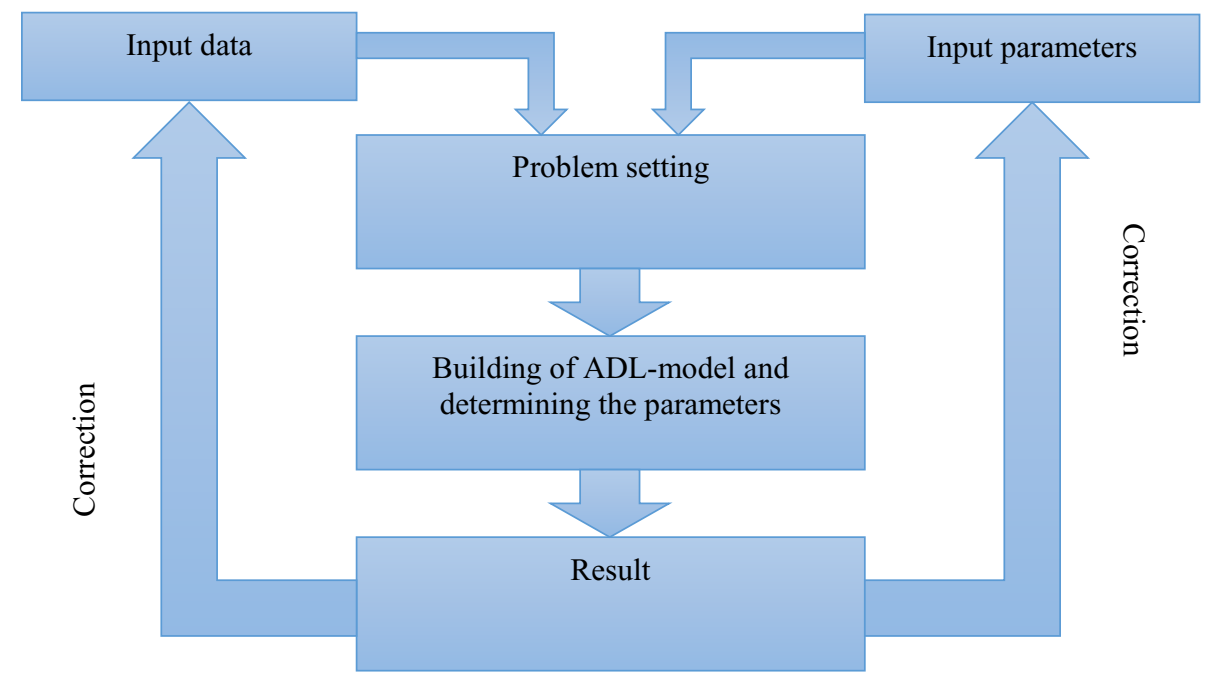

Fig. 3. A variation of recurrent neural network model.

The advantage of the variation is that this model is able to take into account any changes made by the researcher.

\section{Acknowledgements}

This paper is based on research carried out with the financial support of the grant of the Russian Science Foundation (Project No. 14-38- 00009). Peter the Great St. Petersburg Polytechnic University. 


\section{References}

1. G.F. Romashkina, N.I. Didenko, D.F. Skripnuk, Studies on Russian Economic Development 28(1), 22-30 (2017)

2. D.A. Tarkhov, A.N. Vasilyev, Nonlin. Phenom. Complex Syst. 17(3), 327-335 (2014)

3. N.U. Kainov, D.A. Tarkhov, T.A. Shemyakina, Nonlin. Phenom. Complex Syst. 17(1), 57-63 (2014)

4. S.A. Atroshenko, I.A. Korolyov, N. Didenko, Materials Physics and Mechanics 26(1), 26-29 (2016)

5. D.Y. Rudenko, T.V. Pogodaeva, N.I. Didenko, Mediterranean Journal of Social Sciences 6(1), 32 -39 (2015)

6. N. Didenko, K. Kunze, WIT Transactions on Ecology and the Environment 186, 3-11 (2014)

7. N. Didenko, D. Rudenko, D. Skripnuk, International Multidisciplinary Scientific GeoConference Surveying Geology and Mining Ecology Management, SGEM III 3(5), 267-274 (2015)

8. N. Didenko, K. Kunze, D. Skripnuk, Mediterranean Journal of Social Sciences 6(5S2), 473-481 (2015)

9. N. Didenko, D. Skripnuk, O. Krasulina, International Multidisciplinary Scientific Conference on Social Sciences and Arts V, 471-478 (2016)

10. M. Radion, N. Didenko, D. Zenchenko, International Multidisciplinary Scientific Conferences on Social Sciences and Arts, SGEM 2016 V, 817-824 (2016)

11. D. Rudenko, N. Didenko, WELLSO 2015 - II International Scientific Symposium on Lifelong Wellbeing in the World, European Proceedings of Social and Behavioural Scienes 7, 514-521 (2015)

12. N. Didenko, D. Skripnuk, WIT Transactions on Ecology and the Environment 190(1), 151-159 (2014)

13. D. Rudenko, N. Didenko, Proceedings of the 3rd International Conference on European Integration, 813-822 (2016)

14. A. Krogh, J. Vedelsby, Neural Network Ensembles, Cross Validation, and Active Learning (Advances in neural information processing, 1995)

15. E.M. Azoff, Neural Network Time Series Forecasting of Financial Markets (John Wiley \& Sons, Inc. New York, 1994) 